\title{
ESSENTIAL SPECTRAL RADIUS OF QUASICOMPACT ENDOMORPHISMS OF LIPSCHITZ ALGEBRAS
}

\author{
A. GOLBAHARAN AND H. MAHYAR
}

\begin{abstract}
We establish a formula for the essential spectral radius of an endomorphism $T$ of Lipschitz algebras under a condition which is equivalent to the quasicompactness of the endomorphism $T$. We also conclude a necessary and sufficient condition for an endomorphism of these algebras to be Riesz. Finally, we get a relation for the spectrum and the set of eigenvalues of a quasicompact and Riesz endomorphism of these algebras.
\end{abstract}

1. Introduction. Let $(X, d)$ be a compact metric space with infinitely many points and $0<\alpha \leq 1$. The Lipschitz algebra of order $\alpha$, $\operatorname{Lip}(X, \alpha)$, is the algebra of all complex-valued functions $f$ on $X$ for which

$$
p_{\alpha}(f)=\sup \left\{\frac{|f(x)-f(y)|}{d^{\alpha}(x, y)}: x, y \in X \quad \text { and } \quad x \neq y\right\}<\infty .
$$

The subalgebra of those functions $f$ with

$$
\lim _{d(x, y) \rightarrow 0} \frac{|f(x)-f(y)|}{d^{\alpha}(x, y)}=0,
$$

is denoted by $\operatorname{lip}(X, \alpha)$. These Lipschitz algebras were first studied by Sherbert $[\mathbf{1 2}, \mathbf{1 3}]$. The algebras $\operatorname{Lip}(X, \alpha)$ for $0<\alpha \leq 1$ and $\operatorname{lip}(X, \alpha)$ for $0<\alpha<1$ are natural Banach function algebras on $X$ under the norm $\|f\|_{\alpha}=\|f\|_{X}+p_{\alpha}(f)$, where $\|f\|_{X}=\sup _{x \in X}|f(x)|$. Recall that a function algebra $A$ on a compact Hausdorff space $X$ is called natural if every nonzero complex homomorphism on $A$ is an evaluation homomorphism at some point of $X$ [3, Definition 4.1.3]. We note that $\operatorname{Lip}(X, 1) \subseteq \operatorname{lip}(X, \alpha) \subseteq \operatorname{Lip}(X, \alpha)($ see $[\mathbf{1}, \mathbf{7}])$.

2010 AMS Mathematics subject classification. Primary 46J10, Secondary 47B48, 47B38.

Keywords and phrases. Essential norm, essential spectral radius, spectrum, quasicompact endomorphisms, Riesz endomorphisms, Lipschitz algebras.

Received by the editors on October 13, 2012, and in revised form on August 9, 2013.

DOI: $10.1216 /$ RMJ-2015-45-4-1149 
It is known that, if $A$ is a natural Banach function algebra on a compact Hausdorff space $X$ and $T$ is a unital endomorphism of $A$, then there exists a self-map $\varphi$ on $X$ such that $T f=f \circ \varphi$ for all $f \in A$. The converse does not hold in general. That is, given a continuous self-map $\varphi: X \rightarrow X$, the mapping $T$ defined on $A$ by $T f=f \circ \varphi$ does not in general take $A$ into $A$. However, if $\varphi$ is a self-map on $X$ such that, for every $f \in A, f \circ \varphi \in A$, then $T: f \mapsto f \circ \varphi$ is a unital endomorphism of $A$. In each case, we say that $T$ is induced by $\varphi$. Thus, any unital endomorphism $T$ of $A$ can be regarded as a composition operator $C_{\varphi}$, and conversely any composition operator on $A$ is a unital endomorphism. Sherbert in [12, Theorem 5.1] showed that a linear map $T$ on $\operatorname{Lip}(X, \alpha)$ is a unital endomorphism if and only if there exists a self-map $\varphi: X \rightarrow X$ such that $T f=f \circ \varphi$ for all $f \in \operatorname{Lip}(X, \alpha)$ and $d(\varphi(x), \varphi(y)) \leq C d(x, y)$ for some constant $C>0$ and for all $x, y \in X$. In this case, the self-map $\varphi$ is called Lipschitz function, and we write

$$
p(\varphi)=\sup _{x \neq y} \frac{d(\varphi(x), \varphi(y))}{d(x, y)} .
$$

Kamowitz and Shenberg in [8] showed that an endomorphism $T$ of $\operatorname{Lip}(X, \alpha)$ or of $\operatorname{lip}(X, \alpha)$ induced by a self-map $\varphi$ on $X$ is compact if and only if $\varphi$ is a supercontraction, that is,

$$
\lim _{d(x, y) \rightarrow 0} \frac{d(\varphi(x), \varphi(y))}{d(x, y)}=0 .
$$

In this note, we consider endomorphisms of Lipschitz algebras which are quasicompact or Riesz. For convenience, we give the definition of these notions.

Definition 1.1. Let $E$ be an infinite dimensional Banach space. We denote by $\mathcal{B}(E)$ and $\mathcal{K}(E)$ the Banach algebra of all bounded linear operators and compact linear operators on $E$, respectively. The essential norm $\|T\|_{e}$ of $T \in \mathcal{B}(E)$ is the norm of $T+\mathcal{K}(E)$ in the Calkin algebra $\mathcal{B}(E) / \mathcal{K}(E)$, i.e.,

$$
\|T\|_{e}=\|T-\mathcal{K}(E)\|=\operatorname{dist}(T, \mathcal{K}(E))=\inf \{\|T-K\|: K \in \mathcal{K}(E)\} .
$$

The essential spectral radius $r_{e}(T)$ of $T \in \mathcal{B}(E)$ is given by the formula

$$
r_{e}(T)=\lim _{n \rightarrow \infty}\left(\left\|T^{n}\right\|_{e}\right)^{1 / n}=\lim _{n \rightarrow \infty}\left\|T^{n}-\mathcal{K}(E)\right\|^{1 / n} .
$$


The operator $T \in \mathcal{B}(E)$ is called Riesz if $r_{e}(T)=0$ and quasicompact if $r_{e}(T)<1$.

Clearly, $T$ is compact if and only if its essential norm is zero and $T$ is quasicompact if and only if $\left\|T^{n}\right\|_{e}<1$ for some positive integer $n$. Every Riesz operator is also quasicompact.

Recall that if $T$ is an endomorphism of a Banach function algebra $A$ on $X$ induced by the self-map $\varphi: X \rightarrow X$, then $T^{n}$ is an endomorphism of $A$ induced by the self-map $\varphi_{n}: X \rightarrow X$ for each $n \in \mathbb{N}$, where $\varphi_{n}$ is the $n$th iterate of $\varphi$. We also set $\varphi_{0}=i d$.

Some results have been obtained concerning quasicompact and Riesz endomorphisms of certain Lipschitz subalgebras in $[\mathbf{9}, \mathbf{1 0}$, 11]. Behrouzi [2] studied quasicompact and Riesz endomorphisms of $\operatorname{Lip}(X, \alpha)$ and gave an estimate for the essential spectral radius of an endomorphism of $\operatorname{lip}(X, \alpha)$ under certain conditions. In this note, we assume that $T$ is an endomorphism of $\operatorname{Lipschitz}$ algebras either $\operatorname{Lip}(X, \alpha)$ or $\operatorname{lip}(X, \alpha)$ induced by the self-map $\varphi$ on $X$. We first show that the essential spectral radius of $T$ satisfies

$$
r_{e}(T)=\lim _{n \rightarrow \infty} p\left(\varphi_{n}\right)^{\alpha / n}
$$

when $0<\alpha<1$ and

$$
r_{e}(T) \leq \lim _{n \rightarrow \infty} p\left(\varphi_{n}\right)^{\alpha / n}
$$

when $\alpha=1$, provided $p\left(\varphi_{n_{0}}\right)<1$ for some $n_{0} \in \mathbb{N}$. We conclude that the condition $p\left(\varphi_{n_{0}}\right)<1$ for some $n_{0} \in \mathbb{N}$ is sufficient for the endomorphism $T$ to be quasicompact. Also, this condition is necessary for the quasicompactness of $T$, when $X$ is connected. In addition, we show that $T$ is Riesz if $\lim _{n \rightarrow \infty} p\left(\varphi_{n}\right)^{1 / n}=0$, and this is also a necessary condition provided $X$ is connected and $0<\alpha<1$. We then generalize these results by establishing a formula for the essential spectral radius $r_{e}(T)$ under a condition which is equivalent to the quasicompactness of $T$ without connectedness assumption on $X$. As an immediate consequence of the latter result we obtain a necessary and sufficient condition for the endomorphism $T$ to be Riesz when $0<\alpha<1$. Moreover, when $\alpha=1$, this condition is also sufficient. At the end, using the definition of Riesz point [6, page 217], we get a relation for the spectrum and the set of eigenvalues of a quasicompact and Riesz endomorphism of these algebras. 
2. Results. Let $X$ be a compact metric space with infinitely many points, and let the self-map $\varphi: X \rightarrow X$ be continuous. Then we have a nested sequence $\varphi_{n+1}(X) \subseteq \varphi_{n}(X)$ of nonempty compact sets, whence the intersection $\cap_{n=1}^{\infty} \varphi_{n}(X)$ is also nonempty. Moreover, if $p\left(\varphi_{n}\right) \rightarrow 0$ as $n \rightarrow 0$, then diam $\left(\varphi_{n}(X)\right) \rightarrow 0$; hence, $\cap_{n=1}^{\infty} \varphi_{n}(X)$ is a singleton, say $\left\{x_{0}\right\}$. Using Banach's contraction principle, one can see that $x_{0}$ is the unique fixed point of $\varphi$. Therefore, if one defines the constant function $\theta: X \rightarrow X$ by $\theta(x)=x_{0}$, then

$$
d\left(\varphi_{n}(x), \theta(x)\right)=d\left(\varphi_{n}(x), x_{0}\right)=d\left(\varphi_{n}(x), \varphi_{n}\left(x_{0}\right)\right) \leq p\left(\varphi_{n}\right) \operatorname{diam}(X),
$$

for all $x \in X$. Hence,

$$
\lim _{n \rightarrow \infty} \sup _{x \in X} d\left(\varphi_{n}(x), \theta(x)\right)=\lim _{n \rightarrow \infty} \sup _{x \in X} d\left(\varphi_{n}(x), x_{0}\right)=0,
$$

for some $x_{0} \in X$, if $p\left(\varphi_{n}\right) \rightarrow 0$. Note also that, for $n \in \mathbb{N}$ and $x, y \in X$, with $\varphi_{n}(x) \neq \varphi_{n}(y)$, we have $\varphi_{k}(x) \neq \varphi_{k}(y)$ for each $k=0,1, \ldots, n$ and therefore,

$$
\frac{d\left(\varphi_{n}(x), \varphi_{n}(y)\right)}{d(x, y)}=\prod_{k=1}^{n} \frac{d\left(\varphi_{k}(x), \varphi_{k}(y)\right)}{d\left(\varphi_{k-1}(x), \varphi_{k-1}(y)\right)} \leq p(\varphi)^{n},
$$

from which one obtains $p\left(\varphi_{n}\right) \leq p(\varphi)^{n}$ for all $n \in \mathbb{N}$. It follows that $p\left(\varphi_{n}\right) \rightarrow 0$ if $p(\varphi)<1$. Conversely, if $p\left(\varphi_{n}\right) \rightarrow 0$, then $p\left(\varphi_{n_{0}}\right)<1$ for some positive integer $n_{0}$.

Remark 2.1. Let $(X, d)$ be a compact pointed metric space, that is, a compact metric space with a base point $e \in X$. The Lipschitz space $\operatorname{Lip}_{0}(X, \alpha)$ is the space of all Lipschitz functions $f: X \rightarrow \mathbb{C}$ of order $\alpha(0<\alpha \leq 1)$ which are zero at the base point $e \in X$. The space $\operatorname{Lip}_{0}(X, \alpha)$ is a Banach space under the Lipschitz norm $p_{\alpha}(\cdot)$. The space $\operatorname{lip}_{0}(X, \alpha), 0<\alpha<1$, is the closed subspace consisting of those functions $f \in \operatorname{Lip}_{0}(X, \alpha)$ that satisfy (1.1) (see [15]). Vargas et al. in [14, Theorem 3.1] showed that, if $\varphi: X \rightarrow X$ is a base point preserving Lipschitz mapping, then the essential norm of the composition operator $C_{\varphi}: \operatorname{lip}_{0}(X, \alpha) \rightarrow \operatorname{lip}_{0}(X, \alpha)$ satisfies the lower estimate

$$
\lim _{t \rightarrow 0} \sup _{0<d(x, y)<t} \frac{d(\varphi(x), \varphi(y))^{\alpha}}{d(x, y)^{\alpha}} \leq\left\|C_{\varphi}\right\|_{e} .
$$

Their proof is valid for the $\operatorname{Banach}$ algebras $\operatorname{lip}(X, \alpha)$ and $\operatorname{Lip}(X, \alpha)$ with the norm $\|\cdot\|_{\alpha}$ when $0<\alpha<1$. Using this fact, we obtain a 
formula for the essential spectral radius of a unital endomorphism of Lipschitz algebras.

Considering $p\left(\varphi_{n}\right) \leq p(\varphi)^{n}$, and using the fact that $p\left(\varphi_{m+n}\right) \leq$ $p\left(\varphi_{m}\right) p\left(\varphi_{n}\right), \lim _{n \rightarrow \infty} p\left(\varphi_{n}\right)^{1 / n}$ exists and

$$
\lim _{n \rightarrow \infty} p\left(\varphi_{n}\right)^{1 / n}=\inf _{n} p\left(\varphi_{n}\right)^{1 / n}
$$

(see, for example [3, Proposition A.1.26(iii)]). Therefore, in the next theorem we can replace $\lim _{n \rightarrow \infty} p\left(\varphi_{n}\right)^{1 / n}$ with $\inf _{n} p\left(\varphi_{n}\right)^{1 / n}$.

In the remainder of this paper, we regard $\mathfrak{L}(\alpha)$ as being either the algebra $\operatorname{Lip}(X, \alpha)$ for $0<\alpha \leq 1$ or the algebra $\operatorname{lip}(X, \alpha)$ for $0<\alpha<1$.

Theorem 2.2. Let $X$ be a compact metric space, $0<\alpha<1$ and $T$ an endomorphism of $\operatorname{Lip}(X, \alpha)$ or of $\operatorname{lip}(X, \alpha)$ induced by the selfmap $\varphi$ on $X$. If $p\left(\varphi_{n_{0}}\right)<1$ for some positive integer $n_{0}$, then $r_{e}(T)=\lim _{n \rightarrow \infty} p\left(\varphi_{n}\right)^{\alpha / n}$.

Proof. By Remark 2.1, we have

$$
\lim _{t \rightarrow 0} \sup _{0<d(x, y)<t}\left(\frac{d\left(\varphi_{n}(x), \varphi_{n}(y)\right)}{d(x, y)}\right)^{\alpha} \leq\left\|T^{n}\right\|_{e},
$$

for every $n \in \mathbb{N}$. By the assumption that $p\left(\varphi_{n_{0}}\right)<1$ and by the definition of essential spectral radius $r_{e}(T)=\lim _{n \rightarrow \infty}\left\|T^{n}\right\|_{e}^{1 / n}$, for given $\varepsilon>0$, one can choose a positive integer $j$ such that $p\left(\varphi_{j}\right)<1$ and $\left\|T^{j}\right\|_{e}^{1 / j}<r_{e}(T)+\varepsilon / 2$. Fix a positive integer $j$ with such a property. It follows that

$$
\lim _{t \rightarrow 0} \sup _{0<d(x, y)<t}\left(\frac{d\left(\varphi_{j}(x), \varphi_{j}(y)\right)}{d(x, y)}\right)^{\alpha / j} \leq\left\|T^{j}\right\|_{e}^{1 / j}<r_{e}(T)+\frac{\varepsilon}{2},
$$

and therefore,

$$
\sup _{0<d(x, y)<\delta}\left(\frac{d\left(\varphi_{j}(x), \varphi_{j}(y)\right)}{d(x, y)}\right)^{\alpha / j}<r_{e}(T)+\frac{\varepsilon}{2},
$$

for some $\delta>0$.

Furthermore, $p\left(\varphi_{k j}\right) \leq p\left(\varphi_{j}\right)^{k}<1$ and then $d\left(\varphi_{k j}(x), \varphi_{k j}(y)\right) \leq$ $d(x, y)$ for each $x, y \in X$ and for each positive integer $k$. Let $n \in \mathbb{N}$, 
$x, y \in X$ with $0<d(x, y)<\delta$ and $\varphi_{n j}(x) \neq \varphi_{n j}(y)$. Then $0<$ $d\left(\varphi_{k j}(x), \varphi_{k j}(y)\right)<\delta$ for each $k$, from which we obtain

$$
\begin{aligned}
\left(\frac{d\left(\varphi_{n j}(x), \varphi_{n j}(y)\right)}{d(x, y)}\right)^{\alpha /(n j)} & =\left(\prod_{k=0}^{n-1} \frac{d\left(\varphi_{(k+1) j}(x), \varphi_{(k+1) j}(y)\right)}{d\left(\varphi_{k j}(x), \varphi_{k j}(y)\right)}\right)^{\alpha /(n j)} \\
& =\prod_{k=0}^{n-1}\left(\frac{d\left(\varphi _ { j } \left(\varphi_{k j}(x), \varphi_{j}\left(\varphi_{k j}(y)\right)\right.\right.}{d\left(\varphi_{k j}(x), \varphi_{k j}(y)\right)}\right)^{\alpha /(n j)} \\
& \leq \prod_{k=0}^{n-1} \sup _{0<d(u, v)<\delta}\left(\frac{d\left(\varphi_{j}(u), \varphi_{j}(v)\right)}{d(u, v)}\right)^{\alpha /(n j)} \\
& =\sup _{0<d(x, y)<\delta}\left(\frac{d\left(\varphi_{j}(x), \varphi_{j}(y)\right)}{d(x, y)}\right)^{\alpha / j} \\
& <r_{e}(T)+\frac{\varepsilon}{2} .
\end{aligned}
$$

Therefore,

$$
\sup _{0<d(x, y)<\delta}\left(\frac{d\left(\varphi_{n j}(x), \varphi_{n j}(y)\right)}{d(x, y)}\right)^{\alpha /(n j)} \leq r_{e}(T)+\frac{\varepsilon}{2},
$$

for each $n \in \mathbb{N}$.

Also, since

$$
\lim _{n \rightarrow \infty}\left(r_{e}(T)+\frac{\varepsilon}{2}\right)^{(n-1) / n}=r_{e}(T)+\frac{\varepsilon}{2},
$$

and $\lim _{n \rightarrow \infty} p\left(\varphi_{n j}\right)=0$, there exists $N \in \mathbb{N}$ such that $\left(r_{e}(T)+\right.$ $(\varepsilon / 2))^{(n-1) / n}<r_{e}(T)+\varepsilon$ for every $n \geq N$, and $p\left(\varphi_{N j}\right)<\delta /(\operatorname{diam}(X))$. It follows that

$$
d\left(\varphi_{N j}(x), \varphi_{N j}(y)\right)<\frac{\delta}{\operatorname{diam}(X)} d(x, y) \leq \delta
$$

for each $x, y \in X$.

Let $n>N$ and $x, y \in X$ with $\varphi_{n N j}(x) \neq \varphi_{n N j}(y)$. Then,

$$
\left(\frac{d\left(\varphi_{n N j}(x), \varphi_{n N j}(y)\right)}{d(x, y)}\right)^{\alpha /(n N j)}
$$




$$
\begin{aligned}
& =\left(\frac{d\left(\varphi_{n N j}(x), \varphi_{n N j}(y)\right)}{d\left(\varphi_{N j}(x), \varphi_{N j}(y)\right)} \frac{d\left(\varphi_{N j}(x), \varphi_{N j}(y)\right)}{d(x, y)}\right)^{\alpha /(n N j)} \\
& \leq\left(\frac{d\left(\varphi_{(n-1) N j}\left(\varphi_{N j}(x)\right), \varphi_{(n-1) N j}\left(\varphi_{N j}(y)\right)\right)}{d\left(\varphi_{N j}(x), \varphi_{N j}(y)\right)}\right)^{\alpha /(n N j)} \\
& \leq\left(\sup _{0<d(x, y)<\delta}\left(\frac{d\left(\varphi_{(n-1) N j}(x), \varphi_{(n-1) N j}(y)\right)}{d(x, y)}\right)^{\alpha /[(n-1) N j]}\right)^{(n-1) / n} \\
& <\left(r_{e}(T)+\frac{\varepsilon}{2}\right)^{(n-1) / n}<r_{e}(T)+\varepsilon .
\end{aligned}
$$

Therefore, $p\left(\varphi_{n N j}\right)^{\alpha /(n N j)} \leq r_{e}(T)+\varepsilon$, for each $n>N$. Hence, $\lim _{n \rightarrow \infty} p\left(\varphi_{n}\right)^{\alpha / n}=\inf p\left(\varphi_{n}\right)^{\alpha / n} \leq r_{e}(T)$.

For the converse inequality, using the well-known relations $r_{e}\left(T^{n}\right)=$ $r_{e}(T)^{n}$ and $p\left(\varphi_{n}\right) \leq p(\varphi)^{n}$, one may assume that $n_{0}=1$ and $p(\varphi)<1$. Then $p\left(\varphi_{n}\right) \rightarrow 0$ and $\cap_{n=1}^{\infty} \varphi_{n}(X)=\left\{x_{0}\right\}$, where $x_{0}$ is the unique fixed point of $\varphi$. Define rank one endomorphism $S: \mathfrak{L}(\alpha) \rightarrow \mathfrak{L}(\alpha)$ by $S f=f \circ \theta=f\left(x_{0}\right) 1$ for $f \in \mathfrak{L}(\alpha)$ where $\theta: X \rightarrow X$ is the constant function $\theta(x)=x_{0}$. Let $n \in \mathbb{N}$ and $f \in \mathfrak{L}(\alpha)$ with $\|f\|_{\alpha} \leq 1$. Then,

$$
\begin{aligned}
\left|T^{n} f(x)-S f(x)\right| & =\left|f\left(\varphi_{n}(x)\right)-f\left(x_{0}\right)\right| \leq p_{\alpha}(f) d\left(\varphi_{n}(x), x_{0}\right)^{\alpha} \\
& \leq\|f\|_{\alpha} p\left(\varphi_{n}\right)^{\alpha} d\left(x, x_{0}\right)^{\alpha} \leq p\left(\varphi_{n}\right)^{\alpha}(\operatorname{diam}(X))^{\alpha}
\end{aligned}
$$

for each $x \in X$. Hence, $\left\|T^{n} f-S f\right\|_{X} \leq p\left(\varphi_{n}\right)^{\alpha}(\operatorname{diam}(X))^{\alpha}$. On the other hand,

$$
\begin{aligned}
& \left|\left(T^{n} f-S f\right)(x)-\left(T^{n} f-S f\right)(y)\right| \\
& =\left|f\left(\varphi_{n}(x)\right)-f\left(\varphi_{n}(y)\right)\right| \\
& \leq p_{\alpha}(f) d\left(\varphi_{n}(x), \varphi_{n}(y)\right)^{\alpha} \leq p\left(\varphi_{n}\right)^{\alpha} d(x, y)^{\alpha},
\end{aligned}
$$

for every $x, y \in X$. Thus, $p_{\alpha}\left(T^{n} f-S f\right) \leq p\left(\varphi_{n}\right)^{\alpha}$. Therefore,

$$
\begin{aligned}
\left\|T^{n} f-S f\right\|_{\alpha} & =\left\|T^{n} f-S f\right\|_{X}+p_{\alpha}\left(T^{n} f-S f\right) \\
& \leq\left(1+(\operatorname{diam}(X))^{\alpha}\right) p\left(\varphi_{n}\right)^{\alpha},
\end{aligned}
$$

for all $n \in \mathbb{N}$ and $f \in \mathfrak{L}(\alpha)$ with $\|f\|_{\alpha} \leq 1$. Hence, $\left\|T^{n}-S\right\| \leq$ $\left(1+(\operatorname{diam}(X))^{\alpha}\right) p\left(\varphi_{n}\right)^{\alpha}$ for each $n \in \mathbb{N}$. Therefore,

$$
\left\|T^{n}\right\|_{e}=\left\|T^{n}-\mathcal{K}(\mathfrak{L}(\alpha))\right\| \leq\left\|T^{n}-S\right\| \leq\left(1+(\operatorname{diam}(X))^{\alpha}\right) p\left(\varphi_{n}\right)^{\alpha},
$$


and then,

$$
r_{e}(T)=\lim _{n \rightarrow \infty}\left\|T^{n}\right\|_{e}^{1 / n} \leq \lim _{n \rightarrow \infty} p\left(\varphi_{n}\right)^{\alpha / n}
$$

Considering the last part of the proof of the previous theorem, we note that the converse inequality is true even for $\alpha=1$. In fact, we have the following proposition.

Proposition 2.3. Let $X$ be a compact metric space, and let $T$ be an endomorphism of $\operatorname{Lip}(X, 1)$ induced by the self-map $\varphi$ on $X$. If $p\left(\varphi_{n_{0}}\right)<$ 1 for some positive integer $n_{0}$, then $r_{e}(T) \leq \lim _{n \rightarrow \infty} p\left(\varphi_{n}\right)^{1 / n}$.

It was shown in [2, Theorem 2.1] that an endomorphism $T$ of $\operatorname{Lip}(X, \alpha)$ induced by a self-map $\varphi$ on $X$ is quasicompact if $p\left(\varphi_{n}\right) \rightarrow 0$ and $\varphi_{n}$ converges uniformly on $X$ to the constant function $\theta(x)=x_{0}$ for some $x_{0} \in X$. Here, as a consequence of Theorem 2.2 and Proposition 2.3, we obtain that $p\left(\varphi_{n_{0}}\right)<1$ for some $n_{0} \in \mathbb{N}$ is sufficient for an endomorphism $T$ of $\operatorname{Lip}(X, \alpha)(0<\alpha \leq 1)$ or of $\operatorname{lip}(X, \alpha)$ $(0<\alpha<1)$ to be quasicompact (Corollary 2.4 (i)). Also, the function defined in the proof of the converse part of [2, Theorem 2.1] does not belong to $\operatorname{lip}(X, \alpha)$. As Corollary 2.4 (ii), defining a suitable function, a slightly modified argument establishes the converse part of $[\mathbf{2}$, Theorem 2.1] for the $\operatorname{Lipschitz}$ algebras $\operatorname{Lip}(X, \alpha)(0<\alpha \leq 1)$ and $\operatorname{lip}(X, \alpha)$ $(0<\alpha<1)$.

Corollary 2.4. Let $X$ be a compact metric space and $T$ an endomorphism of $\operatorname{Lip}(X, \alpha), 0<\alpha \leq 1$ or of $\operatorname{lip}(X, \alpha), 0<\alpha<1$ induced by the self-map $\varphi$ on $X$.

(i) If $p\left(\varphi_{n_{0}}\right)<1$ for some $n_{0} \in \mathbb{N}$, then $T$ is quasicompact.

(ii) If $X$ is connected and $T$ is quasicompact, then $p\left(\varphi_{n_{0}}\right)<1$ for some $n_{0} \in \mathbb{N}$.

Proof.

(i) Let $p\left(\varphi_{n_{0}}\right)<1$ for some $n_{0} \in \mathbb{N}$. Then by Theorem 2.2 and Proposition 2.3, we have

$$
r_{e}(T) \leq \lim _{n \rightarrow \infty} p\left(\varphi_{n}\right)^{\alpha / n}=\lim _{k \rightarrow \infty} p\left(\varphi_{k n_{0}}\right)^{\alpha /\left(k n_{0}\right)} \leq p\left(\varphi_{n_{0}}\right)^{\alpha / n_{0}}<1,
$$

which implies that $T$ is quasicompact. 
(ii) Let $X$ be connected and $T$ be quasicompact. Using [4, Theorem 1.2], there exists $x_{0} \in X$ such that the operators $T^{n}$ converge, in operator norm, to a rank-one endomorphism $S_{0}$ of $\mathfrak{L}(\alpha)$ defined by $S_{0}(f)=f\left(x_{0}\right) 1$. The point $x_{0}$ is the unique fixed point of $\varphi$.

In the case $\alpha=1$, take $\beta=1$; otherwise, choose any $\beta \in(\alpha, 1]$. For each $y \in Y$ and $n \in \mathbb{N}$, define

$$
f_{n}(x)=\frac{d\left(x, \varphi_{n}(y)\right)^{\beta}}{(\operatorname{diam}(X))^{\beta}+(\operatorname{diam}(X))^{\beta-\alpha}},
$$

for $x \in X$. Then $f_{n} \in \mathfrak{L}(\alpha),\left\|f_{n}\right\|_{\alpha} \leq 1$ and

$$
\begin{aligned}
\left\|T^{n}-S_{0}\right\| & \geq\left\|T^{n} f_{n}-S_{0} f_{n}\right\|_{\alpha} \geq p_{\alpha}\left(T^{n} f_{n}-S_{0} f_{n}\right) \\
& =p_{\alpha}\left(f_{n} \circ \varphi_{n}\right) \geq \frac{\left|f_{n} \circ \varphi_{n}(x)-f_{n} \circ \varphi_{n}(y)\right|}{d(x, y)^{\alpha}} \\
& =\frac{1}{(\operatorname{diam}(X))^{\beta}+(\operatorname{diam}(X))^{\beta-\alpha}} \frac{d\left(\varphi_{n}(x), \varphi_{n}(y)\right)^{\beta}}{d(x, y)^{\alpha}},
\end{aligned}
$$

for every $x, y \in X$ with $x \neq y$ and any $\beta \in(\alpha, 1]$ or $\beta=\alpha=1$. Taking limit as $\beta \rightarrow \alpha$, we conclude that

$$
\left\|T^{n}-S_{0}\right\| \geq \frac{1}{(\operatorname{diam}(X))^{\alpha}+1} \frac{d\left(\varphi_{n}(x), \varphi_{n}(y)\right)^{\alpha}}{d(x, y)^{\alpha}},
$$

for every $x, y \in X$ with $x \neq y$. Hence

$$
\begin{aligned}
\left\|T^{n}-S_{0}\right\| & \geq \frac{1}{(\operatorname{diam}(X))^{\alpha}+1} \sup _{x \neq y} \frac{d\left(\varphi_{n}(x), \varphi_{n}(y)\right)^{\alpha}}{d(x, y)^{\alpha}} \\
& =\frac{1}{(\operatorname{diam}(X))^{\alpha}+1} p\left(\varphi_{n}\right)^{\alpha}
\end{aligned}
$$

Therefore, $\lim _{n \rightarrow \infty} p\left(\varphi_{n}\right)=0$ and $p\left(\varphi_{n_{0}}\right)<1$ for some $n_{0} \in \mathbb{N}$.

From the proof of Corollary 2.4, one can obtain the following interesting relation for any endomorphism $T$ of $\mathfrak{L}(\alpha)$ induced by the self-map $\varphi$ on $X$ :

$$
\max \left\{1, \frac{1}{(\operatorname{diam}(X))^{\alpha}+1} p(\varphi)^{\alpha}\right\} \leq\|T\| \leq \max \left\{1, p(\varphi)^{\alpha}\right\} .
$$

In [2, Proposition 2.3], it was shown that an endomorphism $T$ of $\operatorname{Lip}(X, \alpha)$ induced by a self-map $\varphi$ on $X$ is Riesz, if $\lim _{n \rightarrow \infty} p\left(\varphi_{n}\right)^{1 / n}=$ 
0. As an immediate consequence of Theorem 2.2 and Proposition 2.3 one can get this result for the $\operatorname{Lipschitz}$ algebras $\operatorname{Lip}(X, \alpha)$ and $\operatorname{lip}(X, \alpha)$. Also, using Theorem 2.2 and Corollary 2.4 (ii), one can show that the condition $\lim _{n \rightarrow \infty} p\left(\varphi_{n}\right)^{1 / n}=0$ is necessary for the endomorphism $T$ of $\operatorname{Lip}(X, \alpha)$ or of $\operatorname{lip}(X, \alpha)$ to be Riesz whenever $0<\alpha<1$ and $X$ is connected.

Corollary 2.5. Let $X$ be a compact metric space, and let $T$ be an endomorphism of $\operatorname{Lip}(X, \alpha)(0<\alpha \leq 1)$ or of $\operatorname{lip}(X, \alpha)(0<\alpha<1)$ induced by the self-map $\varphi$ on $X$.

(i) If $\lim _{n \rightarrow \infty} p\left(\varphi_{n}\right)^{1 / n}=0$, then $T$ is Riesz.

(ii) If $X$ is connected, $0<\alpha<1$ and T is Riesz, then $\lim _{n \rightarrow \infty} p\left(\varphi_{n}\right)^{1 / n}$ $=0$.

In the sequel, we generalize the above obtained results to possibly unconnected metric spaces.

Theorem 2.6. Let $X$ be a compact metric space, $0<\alpha<1$, and $T$ an endomorphism of $\operatorname{Lip}(X, \alpha)$ or of $\operatorname{lip}(X, \alpha)$ induced by the self-map $\varphi$ on $X$. If there exists a decomposition of $X$ into a finite number of mutually disjoint clopen subsets, say $X_{1}, X_{2}, \ldots, X_{m}$, such that, for each $i \in\{1, \ldots, m\}$, there exists $n_{i} \in \mathbb{N}$ with $\varphi_{n_{i}}\left(X_{i}\right) \subseteq X_{i}$ and $p\left(\left.\varphi_{n_{i}}\right|_{X_{i}}\right)<1$, then $r_{e}(T)=\max _{1 \leq i \leq m} \lim _{n \rightarrow \infty} p\left(\left.\varphi_{n n_{i}}\right|_{X_{i}}\right)^{\alpha /\left(n n_{i}\right)}$.

Proof. By Remark 2.1, we have

$$
\begin{aligned}
\lim _{t \rightarrow 0} \sup _{\substack{0<d(x, y)<t \\
x, y \in X_{i}}}\left(\frac{d\left(\varphi_{n}(x), \varphi_{n}(y)\right)}{d(x, y)}\right)^{\alpha} & \leq \lim _{t \rightarrow 0} \sup _{\substack{0<d(x, y)<t \\
x, y \in X}}\left(\frac{d\left(\varphi_{n}(x), \varphi_{n}(y)\right)}{d(x, y)}\right)^{\alpha} \\
& \leq\left\|T^{n}\right\|_{e},
\end{aligned}
$$

for each $i \in\{1,2, \ldots, m\}$ and every $n \in \mathbb{N}$. Similar to the proof of Theorem 2.2 , one can easily deduce that $\lim _{n \rightarrow \infty} p\left(\left.\varphi_{n n_{i}}\right|_{X_{i}}\right)^{\alpha /\left(n n_{i}\right)}=$ $\inf _{n} p\left(\left.\varphi_{n n_{i}}\right|_{X_{i}}\right)^{\alpha /\left(n n_{i}\right)} \leq r_{e}(T)$. Hence,

$$
\max _{1 \leq i \leq m} \lim _{n \rightarrow \infty} p\left(\left.\varphi_{n n_{i}}\right|_{X_{i}}\right)^{\alpha /\left(n n_{i}\right)} \leq r_{e}(T) .
$$

We now show the converse inequality. By the hypotheses, we have $\varphi_{k n_{i}}\left(X_{i}\right) \subseteq X_{i}$ and $p\left(\left.\varphi_{k n_{i}}\right|_{X_{i}}\right)<1$ for each positive integer $k$. There- 
fore, if we set $n_{0}=n_{1} n_{2} \cdots n_{m}$, then $\varphi_{n_{0}}\left(X_{i}\right) \subseteq X_{i}$ and $p\left(\left.\varphi_{n_{0}}\right|_{X_{i}}\right)<1$ for each $i \in\{1,2, \ldots, m\}$. As in the proof of Theorem 2.2, we may assume that $n_{0}=1$, and, in a similar way, we have $\lim _{n \rightarrow \infty} p\left(\left.\varphi_{n}\right|_{X_{i}}\right)=0$ and $\bigcap_{n=1}^{\infty} \varphi_{n}\left(X_{i}\right)=\left\{x_{i}\right\}$ for each $i \in\{1, \ldots, m\}$, where $x_{i} \in X_{i}$ is the unique fixed point of $\left.\varphi\right|_{X_{i}}$. Define the continuous self-map $\theta: X \rightarrow X$ by $\theta(x)=x_{i},\left(x \in X_{i}\right)$ and consider the finite rank endomorphism $S: \mathfrak{L}(\alpha) \rightarrow \mathfrak{L}(\alpha)$ by $S f=f \circ \theta=\sum_{i=1}^{m} f\left(x_{i}\right) \chi_{X_{i}}$, where $\chi_{X_{i}}$ is the characteristic function of $X_{i}$.

Let $n \in \mathbb{N}$ and $f \in \mathfrak{L}(\alpha)$ with $\|f\|_{\alpha} \leq 1$. Then

$$
\left\|T^{n} f-S f\right\|_{X} \leq(\operatorname{diam}(X))^{\alpha} \max _{1 \leq i \leq m} p\left(\left.\varphi_{n}\right|_{X_{i}}\right)^{\alpha} .
$$

Set $\mu=\min _{1 \leq i<j \leq m} d\left(X_{i}, X_{j}\right)$. Then

$$
\frac{\left|\left(T^{n} f-S f\right)(x)-\left(T^{n} f-S f\right)(y)\right|}{d(x, y)^{\alpha}} \leq \max _{1 \leq i \leq m} p\left(\left.\varphi_{n}\right|_{X_{i}}\right)^{\alpha},
$$

when $x, y$ belong to the same $X_{i}$, and

$$
\frac{\left|\left(T^{n} f-S f\right)(x)-\left(T^{n} f-S f\right)(y)\right|}{d(x, y)^{\alpha}} \leq \frac{2}{\mu^{\alpha}}(\operatorname{diam}(X))^{\alpha} \max _{1 \leq i \leq m} p\left(\left.\varphi_{n}\right|_{X_{i}}\right)^{\alpha},
$$

when $x, y$ are in the different $X_{i}$. Hence,

$$
p_{\alpha}\left(T^{n} f-S f\right) \leq\left(1+\frac{2}{\mu^{\alpha}}(\operatorname{diam}(X))^{\alpha}\right) \max _{1 \leq i \leq m} p\left(\left.\varphi_{n}\right|_{X_{i}}\right)^{\alpha} .
$$

Therefore,

$$
\left\|T^{n}-S\right\| \leq\left(1+\left(\frac{2}{\mu^{\alpha}}+1\right)(\operatorname{diam}(X))^{\alpha}\right) \max _{1 \leq i \leq m} p\left(\left.\varphi_{n}\right|_{X_{i}}\right)^{\alpha},
$$

for each $n \in \mathbb{N}$. Whence,

$$
\left\|T^{n}\right\|_{e} \leq\left(1+\left(\frac{2}{\mu^{\alpha}}+1\right)(\operatorname{diam}(X))^{\alpha}\right) \max _{1 \leq i \leq m} p\left(\left.\varphi_{n}\right|_{X_{i}}\right)^{\alpha},
$$

and then $r_{e}(T) \leq \max _{1 \leq i \leq m} \lim _{n \rightarrow \infty} p\left(\left.\varphi_{n}\right|_{X_{i}}\right)^{\alpha / n}$.

Remark 2.7. Similar to Proposition 2.3, the inequality

$$
r_{e}(T) \leq \max _{1 \leq i \leq m} \lim _{n \rightarrow \infty} p\left(\left.\varphi_{n n_{i}}\right|_{X_{i}}\right)^{\alpha /\left(n n_{i}\right)}
$$

holds for $\alpha=1$. 
Now we would like to generalize Corollaries 2.4 and 2.5 for possibly unconnected $X$. For this purpose, we shall need the following results due to Feinstein and Kamowitz [5]. We recall that a complex algebra $A$ is semiprime if $J=\{0\}$ is the only ideal in $A$ such that the product of every pair of elements in $J$ is 0 . Clearly, Banach function algebras, in particular, Lipschitz algebras, are semiprime.

Lemma 2.8. [5, Lemma 3.1]. Let $B$ be a unital commutative semiprime Banach algebra, and let $T$ be a bounded unital quasicompact endomorphism of B. Suppose that

$$
\sigma(T) \subseteq\{\lambda \in \mathbb{C}:|\lambda|<1\} \cup\{1\},
$$

and that the eigenvalue 1 of $T$ has multiplicity 1 . Then the operators $T^{n}$ converge in operator norm to a rank-one unital endomorphism $S$ of $B$.

Theorem 2.9. [5, Theorem 3.2]. Let $B$ be a unital commutative semiprime Banach algebra, and let $T$ be a bounded unital quasicompact endomorphism of $B$. Then there exists an $n \in \mathbb{N}$ such that $\sigma\left(T^{n}\right) \subseteq$ $\{\lambda \in \mathbb{C}:|\lambda|<1\} \cup\{1\}$. For such $n$, the unital quasicompact endomorphism $T^{n}$ of $B$ has the following properties:

(i) The eigenspace of $T^{n}$ corresponding to eigenvalue 1 is a finite dimensional, unital subalgebra of $B$ isomorphic to $\mathbb{C}^{m}$ for some $m \in \mathbb{N}$, and hence spanned by $m$ orthogonal idempotents, say $e_{1}, e_{2}, \ldots, e_{m}$.

(ii) Set $B_{i}=e_{i} B(1 \leq i \leq m)$. Then (under an equivalent norm) each $B_{i}$ is a commutative, unital semiprime Banach algebra, with identity $e_{i}$, and

$$
B=\bigoplus_{i=1}^{m} B_{i}
$$

(iii) For $1 \leq i \leq m,\left.T^{n}\right|_{B_{i}}$ is a unital quasicompact endomorphism of $B_{i}$, and $\left.T^{n}\right|_{B_{i}}$ satisfies the conditions of Lemma 2.8. The operators $\left\{\left.T^{k n}\right|_{B_{i}}\right\}_{k=1}^{\infty}$ converge in operator norm to a rank-1 unital endomorphism of $B_{i}$, say $S_{i}$.

(iv) The operators $\left\{T^{k n}\right\}_{k=1}^{\infty}$ converge in operator norm to the rank-m 
endomorphism $S$ of $B$ given by

$$
S(b)=\sum_{i=1}^{m} S_{i}\left(b e_{i}\right) \quad(b \in B)
$$

We are now in a position to prove the generalization of Corollaries 2.4 and 2.5 .

Theorem 2.10. Let $X$ be a compact metric space and $T$ be an endomorphism of $\operatorname{Lip}(X, \alpha), 0<\alpha \leq 1$, or of $\operatorname{lip}(X, \alpha), 0<\alpha<1$ induced by the self-map $\varphi$ on $X$. Then $T$ is quasicompact if and only if there exists a decomposition of $X$ into a finite number of mutually disjoint clopen subsets, say $X_{1}, X_{2}, \ldots, X_{m}$ such that, for each $i \in\{1,2, \ldots, m\}$, there exists $n_{i} \in \mathbb{N}$ with $\varphi_{n_{i}}\left(X_{i}\right) \subseteq X_{i}$ and $p\left(\left.\varphi_{n_{i}}\right|_{X_{i}}\right)<1$.

Proof. If there exists a decomposition of $X$ with such properties in the statement, then by Theorem 2.6 and Remark 2.7, $r_{e}(T) \leq$ $\max _{1 \leq i \leq m} \lim _{n \rightarrow \infty} p\left(\left.\varphi_{n n_{i}}\right|_{X_{i}}\right)^{\alpha /\left(n n_{i}\right)}<1$. Hence, $T$ is quasicompact.

Conversely, suppose that $T$ is quasicompact. By Theorem 2.9 (i), there exists $n_{0} \in \mathbb{N}$ such that $\left\{f: T^{n_{0}} f=f\right\}=\left\{f: f \circ \varphi_{n_{0}}=f\right\}$ is a finite dimensional, unital subalgebra of $\mathfrak{L}(\alpha)$ spanned by $m$ orthogonal idempotents, say $e_{1}, e_{2} \ldots, e_{m}$. Therefore, there exists a finite number of mutually disjoint clopen subsets of $X$, say $X_{1}, X_{2} \ldots, X_{m}$ with union $X$ and

$$
\left\{f: T^{n_{0}} f=f\right\}=\left\{f: f \circ \varphi_{n_{0}}=f\right\}=\left\{\sum_{i=1}^{m} \lambda_{i} \chi_{x_{i}}: \lambda_{1}, \lambda_{2}, \ldots, \lambda_{m} \in \mathbb{C}\right\} .
$$

Then $\varphi_{n_{0}}\left(X_{i}\right) \subseteq X_{i}$ for each $i \in\{1,2, \ldots, m\}$.

Set $\mathfrak{L}_{i}(\alpha)=\chi_{X_{i}} \mathfrak{L}(\alpha)$. In fact, either $\mathfrak{L}_{i}(\alpha) \simeq \operatorname{Lip}\left(X_{i}, \alpha\right)$ for $0<\alpha \leq 1$ or $\mathfrak{L}_{i}(\alpha) \simeq \operatorname{lip}\left(X_{i}, \alpha\right)$ for $0<\alpha<1$. Also, by Theorem 2.9 (iii), $\left.T^{n_{0}}\right|_{\mathfrak{L}_{i}(\alpha)}$ is a quasicompact endomorphism of $\mathfrak{L}_{i}(\alpha)$ induced by the self-map $\left.\varphi_{n_{0}}\right|_{X_{i}}$, for each $i \in\{1,2, \ldots, m\}$, and the operators $\left\{\left.T^{n n_{0}}\right|_{\mathfrak{L}_{i}(\alpha)}\right\}_{n=1}^{\infty}$ converge, in operator norm, to a rank-1 unital endomorphism of $\mathfrak{L}_{i}(\alpha)$, say $S_{i}$. Since $S_{i}$ is a rank-1 unital endomorphism of $\mathfrak{L}_{i}(\alpha)$, there exists $x_{i} \in X_{i}$ such that $S_{i}\left(\left.f\right|_{X_{i}}\right)=$ $f\left(x_{i}\right) 1$ for $f \in \mathfrak{L}(\alpha)$, similar to the proof of Corollary 2.4, one can show 
that

$$
\left\|T^{n n_{0}}-S_{i}\right\| \geq \frac{1}{\left(\operatorname{diam}\left(X_{i}\right)\right)^{\alpha}+1} p\left(\left.\varphi_{n n_{0}}\right|_{X_{i}}\right)^{\alpha} .
$$

Therefore, $\lim _{n \rightarrow \infty} p\left(\left.\varphi_{n n_{0}}\right|_{X_{i}}\right)=0$ and $p\left(\left.\varphi_{n_{i}}\right|_{X_{i}}\right)<1$ for some $n_{i} \in$ $\mathbb{N}$.

Corollary 2.11. Let $X$ be a compact metric space and $T$ be an endomorphism of $\operatorname{Lip}(X, \alpha), 0<\alpha \leq 1$ or of $\operatorname{lip}(X, \alpha), 0<\alpha<1$ induced by the self-map $\varphi$ on $X$. Then, for $0<\alpha<1, T$ is Riesz if and only if there exists a decomposition of $X$ into a finite number of mutually disjoint clopen subsets, say $X_{1}, X_{2}, \ldots, X_{m}$ such that, for each $i \in\{1,2, \ldots, m\}$, there exists $n_{i} \in \mathbb{N}$ with $\varphi_{n_{i}}\left(X_{i}\right) \subseteq X_{i}$ and $\lim _{n \rightarrow \infty} p\left(\varphi_{n n_{i}} \mid X_{i}\right)^{1 / n}=0$. Moreover, when $\alpha=1$, these conditions also imply that $T$ is Riesz.

Proof. If there exists a decomposition of $X$ with such properties, then one can say, $\lim _{n \rightarrow \infty} p\left(\left.\varphi_{n n_{i}}\right|_{X_{i}}\right)=0$, whence $p\left(\varphi_{n n_{i}} \mid X_{i}\right)<1$ for some $n \in \mathbb{N}$. Then using Theorem 2.6, Remark 2.7 and the hypothesis, we have $r_{e}(T) \leq \max _{1 \leq i \leq m} \lim _{n \rightarrow \infty} p\left(\left.\varphi_{n n_{i}}\right|_{X_{i}}\right)^{\alpha / n}=0$ which implies that $T$ is Riesz.

Conversely, suppose that $T$ is a Riesz endomorphism. Then it is also quasicompact and $r_{e}(T)=0$. Therefore, using Theorems 2.6 and 2.10, the result is concluded.

We conclude this paper by establishing some results about $\sigma(T)$ the spectrum of $T$ and $\sigma_{p}(T)$ the set of eigenvalues of $T$.

Theorem 2.12. Let $X$ be a compact metric space, $0<\alpha<1$ and $T$ be a quasicompact endomorphism of $\operatorname{Lip}(X, \alpha)$ or of $\operatorname{lip}(X, \alpha)$ induced by the self-map $\varphi$ on $X$. Then

$$
\begin{gathered}
\sigma_{p}(T) \subseteq\left\{\lambda:|\lambda| \leq r_{e}(T)\right\} \cup\left\{\lambda: \lambda^{n}=1\right\}, \\
\sigma(T) \subseteq\left\{\lambda:|\lambda| \leq r_{e}(T)\right\} \cup\left\{\lambda: \lambda^{n}=1\right\},
\end{gathered}
$$

for some positive integer $n$. In particular, 1 is an isolated point of the spectrum of $T$. 
Proof. According to the proof of Theorem 2.10 there exist positive integer $n$ and a finite number of mutually disjoint clopen subsets of $X$, say $X_{1}, X_{2} \ldots, X_{m}$, with union $X$ such that $\varphi_{n}\left(X_{i}\right) \subseteq X_{i}$ and $\lim _{k \rightarrow \infty} p\left(\varphi_{\left.k n\right|_{X_{i}}}\right)=0$, and so there is the unique fixed point of $\varphi_{\left.n\right|_{X_{i}}}$ say $x_{i}$, for each $i \in\{1,2, \ldots, m\}$. Take any $\lambda \in \mathbb{C}$ with $\lambda^{n} \neq 1$. For each $f \in \operatorname{ker}(\lambda I-T)$, we have $f \circ \varphi=\lambda f$, and then $f\left(x_{i}\right)=f \circ \varphi_{n}\left(x_{i}\right)=\lambda^{n} f\left(x_{i}\right)$, which implies $f\left(x_{i}\right)=0$ for each $i \in\{1,2, \ldots, m\}$. If $f$ is non-zero then there exists a point $x \in X_{i}$ for some $i \in\{1,2, \ldots, m\}$ such that $f(x) \neq 0$ and, for each positive integer $k$,

$$
\left|\lambda^{k n} f(x)\right|=\left|f \circ \varphi_{k n}(x)-f \circ \varphi_{k n}\left(x_{i}\right)\right| \leq d\left(x, x_{i}\right)^{\alpha} p_{\alpha}(f) p\left(\left.\varphi_{k n}\right|_{X_{i}}\right)^{\alpha},
$$

and then

$$
|\lambda||f(x)|^{1 /(k n)} \leq(\operatorname{diam}(X))^{\alpha} p_{\alpha}(f)^{1 /(k n)}\left(p\left(\left.\varphi_{k n}\right|_{X_{i}}\right)\right)^{\alpha /(k n)} .
$$

Taking the limit as $k \rightarrow \infty$,

$$
\left.|\lambda| \leq \lim _{k \rightarrow \infty} p\left(\varphi_{k n} \mid X_{i}\right)\right)^{\alpha /(k n)} \leq r_{e}(T) .
$$

Hence, for each $\lambda \in \mathbb{C}$ with $\lambda^{n} \neq 1$, if $|\lambda|>r_{e}(T)$, then $\operatorname{ker}(\lambda I-T)=$ $\{0\}$, which implies (2.1).

Moreover, if $|\lambda|>r_{e}(T)$, then also $\operatorname{ker}(\lambda I-T)=\{0\}$. Using [6, Propositions 51.8 and 52.1], if $|\lambda|>r_{e}(T)$, then $\lambda$ is a Riesz point of $T$, and, by the definition of the Riesz point [6, page 217], the operator $\lambda I-T$ is invertible, that is, $\lambda \notin \sigma(T)$. Therefore, the relation (2.2) follows.

As an immediate consequence, we have

Corollary 2.13. Let $X$ be a compact metric space, $0<\alpha<1$, and $T$ an endomorphism of $\operatorname{Lip}(X, \alpha)$ or of $\operatorname{lip}(X, \alpha)$ induced by the self-map $\varphi$ on $X$.

(i) If $T$ is Riesz, then $\sigma(T) \subseteq\{0\} \cup\left\{\lambda: \lambda^{n}=1\right\}$ for some positive integer $n$.

(ii) If $T$ is quasicompact and $\sigma(T) \subseteq\{0\} \cup\{\lambda:|\lambda|=1\}$, then $T$ is Riesz. 
We remark that, in Theorem 2.12 and Corollary 2.13, if we assume the connectedness of $X$, we get $n=1$. Therefore, by Corollary 2.13, if $X$ is a connected compact metric space and the endomorphism $T$ is Riesz, then $\sigma(T)=\{0,1\}$.

\section{REFERENCES}

1. W.G. Bade, P.C. Curtis, Jr., and H.G. Dales, Amenability and weak amenability for Beurling and Lipschitz algebras, Proc. Lond. Math. Soc. 55 (1987), 359-377.

2. F. Behrouzi, Riesz and quasi-compact endomorphisms of Lipschitz algebras, Houst. J. Math. 36 (2010), 793-802.

3. H.G. Dales, Banach algebras and automatic continuity, Lond. Math.Soc. Mono. 24, The Clarendon Press, Oxford, 2000.

4. J.F. Feinstein and H. Kamowitz, Quasicompact and Riesz endomorphisms of Banach algebras, J. Funct. Anal. 225 (2005), 427-438.

5. __ Quasicompact endomorphisms of commutative semiprime Banach algebras, Banach Center Publ. 91 (2010), 159-167.

6. H.G. Heuser, Functional analysis, John Wiley and Sons, New York, 1982.

7. T.G. Honary and H. Mahyar, Approximation in Lipschitz algebras, Quaest. Math. 23 (2000), 13-19.

8. H. Kamowitz and S. Scheinberg, Some properties of endomorphisms of Lipschitz algebras, Stud. Math. 24 (1990), 383-391.

9. H. Mahyar, Quasicompact and Riesz endomorphisms of infinitely differentiable Lipschitz algebras, Southeast Asian Bull. Math. 35 (2011), 249-259.

10. H. Mahyar and A. H. Sanatpour, Quasicompact endomorphisms of Lipschitz algebras of analytic functions, Publ. Math. Debr, 76 (2010), 135-145.

11. Compact and quasicompact homomorphisms between differentiable Lipschitz algebras, Bull. Belg. Math. Soc. Simon-Stevin 17 (2010), 485-497.

12. D.R. Sherbert, Banach algebras of Lipschitz functions, Pac. J. Math. 13 (1963), 1387-1399.

13. The structure of ideals and point derivations in Banach algebras of Lipschitz functions, Trans. Amer. Math. Soc. 111 (1964), 240-272.

14. A.J. Vargas, M. Lacruz and M.V. Vallecillos, Essential norm of composition operators on Banach spaces of Hölder functions, Hindawi Publishing Corporation, Abstract and Applied Analysis (2011), Article ID 590853, 13 pages.

15. N. Weaver, Lipschitz algebras, World Scientific, Singapore, 1999.

Department of Mathematics, Kharazmi University (Tarbiat Moallem University), 50, Taleghani Ave., 15618, Tehran Iran

Email address: golbaharan_azin@yahoo.com, golbaharan@khu.ac.ir

Department of Mathematics, Kharazmi University (Tarbiat Moallem University), 50, Taleghani Ave., 15618, Tehran Iran

Email address: mahyar@khu.ac.ir 